\title{
CD42 Complex
}

National Cancer Institute

\section{Source}

National Cancer Institute. CD42 Complex. NCI Thesaurus. Code C126122.

A heterotetrameric protein complex expressed on the surface of platelets that is involved in promoting platelet adhesion to von Willebrand factor during wound healing. This noncovalent complex is comprised of glycoprotein 5 (GPV; CD42d), glycoprotein 9 (GPIX; CD42a), and glycoprotein Ib (GPIb). GPIb is a heterodimer comprised of platelet glycoprotein Ib alpha chain (GPIba; CD42b) covalently bound to platelet glycoprotein Ib beta chain (GPIbb; CD42c). 